\title{
EFECTO DEL TUTORADO Y DISTANCIAS DE SIEMBRA SOBRE EL RENDIMIENTO DE Physalis peruviana $L$.
}

\section{EFFECT OF PLANT DENSITY AND TRAINING SYSTEMS ON YIELD OF Physalis peruviana $L$.}

\author{
Enrique Quevedo García ${ }^{1}$, Orlando Sánchez García ${ }^{2}$, Carlos Eliécer Veloza Sandoval ${ }^{3}$
}

\begin{abstract}
${ }^{1}$ Ing. Agrónomo, M.Sc., Profesor Asociado. Universidad de Pamplona, Facultad de Ciencias Agrarias, Departamento de Agronomía. km 1 vía Bucaramanga El Buque, Pamplona, Norte de Santander, Colombia, e-mail: enriquegarcia@unipamplona. edu.co; ${ }^{2}$ Ing. Agrónomo, Esp. Gerencia Técnica y de Mercadeo Agromil-Bioest, e-mail: orlando.sanchez@bioest.com.co; ${ }^{3}$ Ing. Agrónomo. Ing. Administrador Palmeras Inveragro Báez, e-mail: cvelozasandoval@yahoo.es
\end{abstract}

Rev. U.D.C.A Act. \& Div. Cient. 18(1): 91-99, Enero-Junio, 2015

\section{RESUMEN}

Se estableció un experimento en una finca productora de uchuva en Subia, Cundinamarca, con el objetivo de evaluar tres sistemas de tutorado tipo espaldera, chiquero, escalera y tradicional y tres distancias de siembra, de $1 \times 2$, 2x2 y 3x2m, con el fin de establecer el mejor tratamiento que combine el tutor y la distancia de siembra, que mejore los rendimientos. El ensayo fue llevado a cabo durante un año. En este lapso, se midieron variables morfológicas de rendimiento y de calidad del fruto. Se empleó un diseño de parcelas divididas, donde la parcela principal estuvo compuesta por los tipos de tutorado y, la parcela secundaria, por las distancias de siembra. Se usaron tres repeticiones, con una unidad experimental cambiante, según la densidad evaluada. Las variables altura de la planta, numero de ramas, área foliar, índice de área foliar, numero de flores, diámetro longitudinal, volumen del fruto, número de frutos, rendimiento por planta y rendimiento por hectárea presentaron diferencias significativas para la interacción entre los tutores y las densidades. Se concluyó, que el sistema de tutorado en chiquero y la distancia de siembra $1 \times 2 \mathrm{~m}$, específicamente, son los más aconsejables para las condiciones experimentales del ensayo, de acuerdo a la significancia del rendimiento por planta y rendimiento por hectárea obtenidos.

Palabras clave: Uchuva, cosecha, componentes de rendimiento.

\section{SUMMARY}

An experiment was carried out at the Cape gooseberry production farm in Subia, Cundinamarca, to evaluate three training espalier systems, "chiquero", staircase and traditional and three planting density, $1 \times 2,2 \times 2$ y $3 \times 2 m$, in order to establish the best treatment which combines training systems and planting distance for yield improvement. The research was carried out during one year. During this time variables of morphological aspect, yield and fruit quality were measured. An experimental design of split plots was employed, where the main plot constituted the three training systems and the secondary plots the planting density. Three repetitions were used, with a changing experimental unit according to the evaluated distances. The variables plant height, number of branches, leaf area index, leaf area, number of flowers, longitudinal diameter, fruit volume, number fruit, yield per plant and per hectare, showed significant differences for the interaction between training systems and planting density. It was concluded that the treatments with the "chiquero" tutoring system and planting at the distance of $1 \times 2 \mathrm{~m}$ are the most desirable ones under the experimental conditions, according to the significance of yield per plant per hectare obtained.

Key words: Cape gooseberry, harvest, yield components.

\section{INTRODUCCIÓN}

La uchuva es una planta, cuyas ramas productivas son decumbentes y necesitan un sistema de tutorado (Rufato et al. 2008), para soportarla durante el crecimiento, facilitando las labores culturales y aumentando la productividad (Lima et al. 2009). El tutorado, que se debe usar, está en función de la densidad de siembra, la topografía del terreno, su fertilidad, el ecotipo, el manejo agronómico, las condiciones agroecológicas, la disponibilidad de materiales y sus costos (Fischer \& Miranda, 2012). Sin tutor, la planta puede crecer entre 1,00 a $1,5 \mathrm{~m}$ y con soporte puede exceder los $2 \mathrm{~m}$ de altura (Muniz et al. 2014).

En Colombia, el tutorado en "V" es el más utilizado y consiste en colocar dos repisas de $3 \mathrm{~m}$ de largo, instaladas y en- 
terradas en el mismo hueco, formando una V; cada 7m, se coloca otro par de repisas (Fischer \& Miranda, 2012). En Brasil, los principales métodos de tutorado son "X", "V", espaldera simple, "V" invertido, triangular y vertical con bambú (Muniz et al. 2014; Lima et al. 2010; Rufato et al. 2008). Con los actuales sistemas en V, en Colombia, se logran 14t. $\mathrm{ha}^{-1}$, considerado como bajo, si se tiene en cuenta el techo tecnológico de 28t.ha ${ }^{-1}$ (Miranda, 2004).

Las densidades de plantación y el tutor en cada país varían según el sitio agroecológico (como por ejemplo, la topografía), del ecotipo (variedad), de la fitosanidad y del manejo del cultivo (Fischer et al. 2014). En Brasil, las distancias de siembra del cultivo varían, entre plantas, de 0,50 a 1,50m, de acuerdo al tutor. Se recomienda las distancias de 1,00x3,00 o 0,50x3,00m entre plantas y entre surcos, respectivamente (Muniz et al. 2014). En la sabana de Bogotá (Colombia), se siembra a 1x2m y en Subia a 2,0x2,5m (Ramírez et al. 2013). En Bulgaria, la distancia que se usa es de $0,5 \times 0,7 \mathrm{~m}$, en variedad Plovdiv y Obrazec 1 Ecotipo Colombia (Panayotov \& Popova, 2013). En la India, Ali \& Singh (2014) manejaron dis-

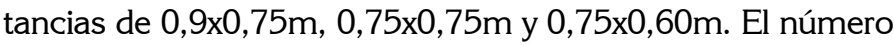
de plantas por hectárea determina la densidad de siembra y el monto de los costos del cultivo (Lima et al. 2009).
En las zonas altas se recomienda sembrar con una mayor densidad, lo que hace necesario un raleo de cada segunda planta cuando se haya terminado el primer pico de producción (Angulo, 2005a; Fischer \& Miranda, 2012).

La investigación en tres tipos de espaldera tuvo como fin establecer el tipo de sistema de conducción, que combinado con la densidad de siembra logrará el mayor rendimiento por hectárea, manteniendo la calidad de la producción.

\section{MATERIALES Y MÉTODOS}

Localización: El ensayo, se realizó durante el 2003 en Subia, Municipio de Silvania (Cundinamarca), a $7^{\circ} 31^{\prime} 37,5^{\prime \prime}$, de latitud norte y $74^{\circ} 22^{\prime} 40,1^{\prime \prime}$, de longitud oeste, a una altura de $2200 \mathrm{msnm}$, temperatura de $16^{\circ} \mathrm{C}$ y precipitación de $1200 \mathrm{~mm}$, situados en el rango de sumamente apto para el cultivo, humedad relativa del $85 \%$ y brillo solar de 2900 horas de luz al año, ubicados en el rango de marginal para el cultivo (Miranda, 2005), con resultados del estudio de las características fisicoquímicas del suelo del lote experimental en Subia, que se muestran en la tabla 1.

De acuerdo al análisis de suelo, se recomendó 30 a 40 días antes del trasplante, incorporar $2 \mathrm{~kg}$ de abono orgánico (Mi-

Tabla 1. Resultados del estudio de las características fisicoquímicas del suelo del lote experimental en Subia, Cundinamarca, antes de la siembra $(0-20 \mathrm{~cm}$ de profundidad).

\begin{tabular}{|l|c|c|}
\hline \multicolumn{2}{|c|}{ Características } & \multicolumn{2}{c|}{ Interpretación } \\
\hline Textura & Franco arenosa & Media a gruesa \\
\hline $\mathrm{pH}$ & 4,99 & Muy fuertemente ácido \\
\hline Materia orgánica (\%) & 13,95 & Bajo \\
\hline Fósforo $\mathrm{ppm}$ & 11,34 & Bajo \\
\hline Calcio me/100g & 4,10 & Bajo \\
\hline Magnesio me/100g & 0,56 & Bajo \\
\hline Potasio me/100g & 0,26 & Bajo \\
\hline Sodio me/100g & 0,26 & Alto \\
\hline Hierro ppm & 291,63 & Óptimo \\
\hline Boro ppm & 0,44 & Alto \\
\hline Cobre ppm & 13,49 & Bajo \\
\hline Manganeso ppm & 3,01 & Deficiencia de Magnesio \\
\hline Relaciones catiónicas entre & & Deficiencia de Magnesio \\
\hline los elementos & & Medio \\
\hline Ca/ $M g$ & 7,32 & Medio \\
\hline Mg/K & 2,15 & \\
\hline Ca/K $/ \mathrm{K}$ & 15,77 & \\
\hline Ca + Mg/K & 17,92 & \\
\hline & & \\
\hline
\end{tabular}


randa, 2004) y 250g de cal dolomítica por hoyo. Se fertilizó, en los siguientes momentos: al trasplante de las plántulas, en crecimiento y en producción, cada dos meses.

Ecotipo estudiado: El material de origen sexual utilizado fue el ecotipo Colombia, obtenido de plantas, en los municipios de Silvania y de Granada, en Cundinamarca.

Diseño experimental: Se empleó un diseño de parcelas divididas en bloques completos al azar, con tres repeticiones, para la evaluación de tres tutores y tres densidades de siembra, en un terreno con una pendiente ligeramente inclinada; el área del experimento fue de $2613,6 \mathrm{~m}^{2}$. Se obtuvieron veintisiete unidades experimentales constituidas por tres tipos de parcelas, debido a las diferencias entre las distancias de siembra; el área de la unidad experimental fue $96,8 \mathrm{~m}^{2}$. El experimento fue montado sobre tres repeticiones. Los tutorados se ubicaron en la parcela principal y, en las subparcelas, las distancias de siembra. La descripción de cada uno de ellos es la siguiente:

El sistema en espaldera tipo chiquero ( $\left.\mathrm{t}_{1}\right)$ constó de dos cuerdas o alambres principales, con dos cuerdas de plástico de ayuda, situadas a menor altura del suelo, con dos cuerdas de alambre No. 12, a 2,00m del suelo y dos cuerdas de fibra plástica, a 1,60m del suelo. El sistema en espaldera tipo escalera ( $\mathrm{t}_{2}$ ) consistió en una espaldera compuesta con alambres a diferente altura, con una primera cuerda de alambre No. 12 , a $2,00 \mathrm{~m}$ del suelo y la segunda, a $1,60 \mathrm{~m}$ del suelo; el sistema en espaldera tipo el tradicional (t3) consistió en un sistema de espaldera compuesta modificado, con dos cuerdas de alambre No. 12, a 2,00m del suelo. En las subparcelas, se ubicaron las distancias de siembra, correspondientes a la distancia $1 \times 2 \mathrm{~m}\left(\mathrm{~d}_{1}\right)$, equivalente a 5.000 plantas.ha $^{-1}$; para la distancia $2 \times 2 \mathrm{~m}\left(\mathrm{~d}_{2}\right)$, equivalente a 2.500 plantas.ha $^{-1}$ y, para la distancia $3 \times 2 \mathrm{~m}\left(\mathrm{~d}_{3}\right)$, equivalente a 1.666 plantas. $\mathrm{ha}^{-1}$. En cada surco, se ubicó un sólo tipo de tutorado; la primera planta, a $50 \mathrm{~cm}$ del borde y después, se ubicaron las otras plantas, a 1, 2 o 3m, según la distancia escogida. La distancia entre surcos, se tomó a partir del tallo principal de cada planta hasta la otra planta, sin importar el tipo de tutor, a 2 o $3 \mathrm{~m}$.

Siembra: Los sitios fueron marcados para el ahoyado y se repicó a una profundidad de $40 \mathrm{~cm}$. Se aplicaron cepas de micorrizas y hongos antagonistas (Trichoderma harzianum y Beauveria bassiana), para el manejo de Fusarium spp. Se desyerbó manualmente durante el periodo crítico de competencia de arvenses.

Variables respuesta: Dos plantas fueron marcadas con cinta amarilla por subparcela para recolectar los datos; la altura, se midió sobre el tallo principal desde el cuello a la yema apical, con un metro plástico; el número de nudos, se contaron teniendo en cuenta el primer nudo visible después del cuello del tallo principal; el diámetro del tallo principal, se midió con un calibrador, a partir de los 20 días de después de la emergencia sobre el tallo, entre el primer y el segundo entrenudo; el número de ramas, se contaron a partir de las generadas del tallo principal, desde 20 días a 305 DDT(días después del trasplante); el número de hojas, se contaron teniendo en cuenta las que estuvieran desarrolladas y en plena función fotosintética, desde los 20 días a 305 DDT; el área foliar por planta, se estimó para una muestra de diez hojas de cada subparcela, usando un integrador de área foliar Licor 3100 y con ese resultado y el número de hojas por planta, se halló el área foliar al momento de la fructificación; el índice de área foliar, se calculó teniendo en cuenta el área foliar por planta y la distancia de siembra; el número de flores, se contaron desde los 29 a 305 DDT; para estimar el volumen del fruto, el peso del fruto y el diámetro longitudinal, se recolectaron diez frutos por subparcela en madurez comercial, tomadas al azar y a los que se les midió el volumen, tomando una probeta con agua y midiendo el desplazamiento de la misma, que provocaron los frutos en $\mathrm{cm}^{3}$; con una balanza, se peso cada fruto; el diámetro longitudinal del fruto, se midió con un calibrador Vernier, teniendo en cuenta de medirlo en el mayor diámetro. Los frutos fueron cosechados desde los 29 a 305 DDT; el rendimiento agronómico por planta, se evalúo con base en el promedio de las dos plantas en plena competencia, marcadas por subparcela, a partir del inicio del primer ciclo productivo; para estimar el rendimiento por hectárea, se utilizó el rendimiento por planta y se calculó con la distancia de siembra la producción por hectárea y con un refractómetro manual, se establecieron los grados Brix.

Análisis de la información: Los datos fueron sometidos a un análisis de varianza, para determinar las diferencias entre tutorados, densidades de siembra y su interacción (txd). Se aplicó el programa analítico SAS $®$ versión 9.5. Para analizar los tratamientos producto de la interacción (txd), se usó la prueba de comparación múltiple de medias de Tukey.

\section{RESULTADOS Y DISCUSIÓN}

Altura de la planta, número de nudos, diámetro del tallo y número de ramas: En la tabla 2, se observa que los tratamientos para la altura t3xd1 y t1xd3, con 139,50 y $139,33 \mathrm{~cm}$, respectivamente, se diferenciaron a un nivel de $\mathrm{P}<0,05$ de $\mathrm{t} 2 \mathrm{xd} 3$ con $129,00 \mathrm{~cm}$. Los valores encontrados fueron mayores a los obtenidos en materiales peruanos de Huancayo, sembrados bajo invernadero, en México, por Mora-Aguilar et al. (2006); a los materiales brasileros, sembrados a campo abierto, por Peixoto et al. (2010), fueron similares a los obtenidos en campo abierto en Bulgaria, por Panayotov \& Popova (2014) y menores, a los $233,7 \mathrm{~cm}$, obtenidos en materiales españoles, sembrados bajo condiciones de invernadero de vidrio tipo Venlo, por Torres et al. (2014). 
Los tratamientos $\mathrm{t} 1 \mathrm{xd} 1, \mathrm{t} 3 \mathrm{xd} 1$ y $\mathrm{t} 2 \mathrm{xd} 1$ con mayor número de ramas y altura fueron los de mayores rendimientos por hectárea. El rendimiento por hectárea, se correlacionó, positivamente con la altura de la planta y número de ramas, lo que indicó que la altura de la planta y el número de ramas son factores determinantes de la productividad de la planta, tal como lo hallaron Criollo et al. (2014).
En la tabla 2, se observa un promedio de 10 nudos para los tratamientos, sin qué se diferencien a un nivel de $\mathrm{P}<0,05$. La planta presentó una primera bifurcación natural del eje principal, entre el 8 al 10 nudo, que indujo la fase generativa e, indicó, que el desarrollo de los órganos estuvo regulado por la tasa de aparición de los nudos, como componente central del aumento del índice de área foliar y, por lo tanto, de la

Tabla 2. Valores medios para las variables altura de la planta, numero de nudos, diámetro del tallo y número de ramas, en P. peruviana L.

\begin{tabular}{|c|c|c|c|c|}
\hline Tratamientos & Altura de la planta $(\mathrm{cm})$ & Numero de nudos & Diámetro del tallo $(\mathrm{cm})$ & Número de ramas \\
\hline t1xd1 & $132,67 \mathrm{a} *$ & $10,33 \mathrm{a}$ & $2,25 \mathrm{ab}$ & $89,83 \mathrm{ab}$ \\
\hline $\mathrm{t} 1 \mathrm{xd2}$ & $137,50 \mathrm{ab}$ & $10,50 \mathrm{a}$ & $2,25 \mathrm{ab}$ & $92,17 \mathrm{ab}$ \\
\hline $\mathrm{t} 1 \mathrm{xd} 3$ & $139,30 \mathrm{a}$ & $9,83 \mathrm{a}$ & $2,28 \mathrm{a}$ & $88,17 \mathrm{ab}$ \\
\hline $\mathrm{t} 2 \mathrm{xd} 1$ & $136,50 \mathrm{ab}$ & $10,00 \mathrm{a}$ & $2,22 \mathrm{ab}$ & $96,33 \mathrm{a}$ \\
\hline $\mathrm{t} 2 \mathrm{xd2}$ & $133,00 \mathrm{ab}$ & $10,00 \mathrm{a}$ & $2,23 \mathrm{ab}$ & $83,50 \mathrm{ab}$ \\
\hline t2xd3 & $129,00 \mathrm{~b}$ & $9,83 \mathrm{a}$ & $1,84 \mathrm{~b}$ & $86,83 \mathrm{ab}$ \\
\hline t3xd1 & $139,50 \mathrm{a}$ & $10,83 \mathrm{a}$ & $2,13 \mathrm{ab}$ & $85,00 \mathrm{ab}$ \\
\hline $\mathrm{t} 3 \mathrm{xd2}$ & $130,30 \mathrm{ab}$ & $10,83 \mathrm{a}$ & $2,14 \mathrm{ab}$ & $81,83 \mathrm{~b}$ \\
\hline $\mathrm{t} 3 \mathrm{xd} 3$ & $132,00 \mathrm{ab}$ & $10,17 \mathrm{a}$ & $2,04 \mathrm{ab}$ & $82,83 \mathrm{~b}$ \\
\hline
\end{tabular}

*Medias con la misma letra no difieren estadísticamente, según la prueba de Tukey, $\alpha=0,05$, estadístico de prueba válido cuando la prueba $\mathrm{F}$ resulta no significativa.

intercepción de radiación, el crecimiento del número de ramas, la arquitectura y altura (Salazar et al. 2008). El número de nudos correlacionó con la altura $(0,61)$ y número de ramas $(0,94)$. La tabla 2 muestra que los mejores tratamientos para el diámetro del tallo fueron $\mathrm{t} 1 \mathrm{xd} 3$, con $2,28 \mathrm{~cm}$ y $\mathrm{t} 1 \mathrm{xd} 2$, con $2,254 \mathrm{~cm}$ y se diferenció a un nivel de $\mathrm{P}<0,05$ de $\mathrm{t} 2 \mathrm{xd} 3$ con $1,84 \mathrm{~cm}$, valores mayores a los obtenidos con materiales peruanos de Huancayo, sembrados bajo invernadero, en México (Mora-Aguilar et al. 2006). El menor valor fue hallado en t2xd3 y se debió a la modificación en el tutor de la distancia de siembra, que fue determinante en la respuesta a la competencia entre plantas.

El mejor tratamiento para el número de ramas (Tabla 2) fue t2xd1, con 96,33 ramas y se diferenció a un nivel de $\mathrm{P}<0,05$ de $\mathrm{t} 3 \mathrm{xd} 3$, con 82,83 y t3xd2, con 81,83 . La mayor área de distribución del tallo principal en $\mathrm{t} 2$, al tener los soportes a diferente altura, permitió una óptima penetración de la luz fotosintéticamente activa, que estimuló la aparición de los brotes axilares, al evitar el autosombreamiento, permitiendo el desarrollo de nuevas ramas, hojas, flores y frutos, en forma simultáneamente, como lo reportan Fischer et al. (2011) e, indica, que la productividad de la uchuva está directamente relacionada con el número de ramas (Panayotov \& Popova,
2014). La asociación positiva entre el número de ramas con la altura de planta $(0,90)$ y el número de nudos $(0,60)$ indica que la aparición de las ramas en la planta está supeditada a tener un mínimo número de nudos y una determinada altura de planta.

Número de hojas, área foliar e índice de área foliar: En la tabla 3 , se observa que los tratamientos t1xd2, con 544 hojas, se diferenció a un nivel de $\mathrm{P}<0,05$ de $\mathrm{t} 2 \mathrm{xd} 3$, con 380,50 calculadas durante la fase de fructificación. Los valores fueron mayores a los encontrados en ecotipos peruanos, con 67 (Mora-Aguilar et al. 2006). El número de hojas presentó una asociación con la altura de planta $(0,92)$, diámetro del tallo principal $(0,75)$ y número de ramas $(0,90)$ e, indica, que su óptimo desarrollo está supeditado a alcanzar un mínima altura de planta, número de ramas y diámetro del tallo principal y se alcanzó el mayor número, entre el estadio de floración a fructificación, tal como lo hallaron en Bulgaria, Panayotov \& Popova (2014). Esta característica ha correlacionado positivamente con el rendimiento en trabajos hechos en México, bajo invernadero (Mora-Aguilar et al. 2006).

En la tabla 3, se muestra que los mejores tratamientos para el área foliar fueron $\mathrm{t} 1 \mathrm{xd} 3$, con $2,36 \mathrm{~m}^{2} \mathrm{y} \mathrm{t} 3 \mathrm{xd} 3$, con $1,98 \mathrm{~m}^{2}$ 
y tuvieron diferencias estadísticas a un nivel de $\mathrm{P}<0,05$ con $\mathrm{t} 1 \mathrm{xd} 1$, con $1,23 \mathrm{~m}^{2} \mathrm{e}$, indica, que la menor distancia de siembra ejerce un estrés por competencia ambiental sobre la planta. Se alcanzaron los mayores valores en el estadio de fructificación, tal como se halló en Bulgaria, a campo abierto (Panayotov \& Popova, 2014). Los mejores tratamientos para el IAF fueron $\mathrm{t} 3 \mathrm{xd} 1$, con 16,92; $\mathrm{t} 2 \mathrm{xd} 1$, con 16,47 y t1xd1, con 15,11 (Tabla 3), que corresponden a los de mejor producción por hectárea y que se diferenciaron a un nivel de $\mathrm{P}<0,05$ de los otros tratamientos a los 305DDT, lo que indicó que el IAF se correlacionó positivamente con el rendimiento agronómico por hectárea $(0,55)$.
El uso de sistemas de tutorado en espaldera tipo "chiquero" con cuatro ramas, como el utilizado en Subia, afectó el IAF, ya que presentó limitantes para lograr altos rendimientos con altas densidades de siembra, tal como lo afirma Miranda (2005). Los altos IAFs permitieron que el microambiente en el cultivo no fuera adecuado para la proliferación de plagas, como pulgilla (Epitrix sp.), escarabajo de la papa (Diabrotica sp.), ácaros o arañitas (Aculops sp., Taesonemus sp. y Czenspinskia sp.), pero sí para enfermedades, como marchitamiento vascular (Fusarium sp.) y mancha gris (Cercospora sp.), debido a la baja altitud de Subia, tal como lo reporta Fischer \& Miranda (2012).

Tabla 3. Valores medios para las variables número de hojas, área foliar por planta e índice de área foliar, en P. peruviana L.

\begin{tabular}{|c|c|c|c|}
\hline Tratamientos & Número de hojas & Área foliar por planta $\left(\mathrm{m}^{2}\right)$ & Índice de área foliar \\
\hline $\mathrm{t} 1 \mathrm{xd} 1$ & $419,50 \mathrm{ab} *$ & $1,23 \mathrm{c}$ & $15,11 \mathrm{ab}$ \\
\hline $\mathrm{t} 1 \mathrm{xd} 2$ & $544,00 \mathrm{a}$ & $1,39 \mathrm{bc}$ & $7,53 \mathrm{bc}$ \\
\hline $\mathrm{t} 1 \mathrm{xd} 3$ & $504,50 \mathrm{ab}$ & $2,36 \mathrm{a}$ & $6,51 \mathrm{bcd}$ \\
\hline $\mathrm{t} 2 \mathrm{xd} 1$ & $434,17 \mathrm{ab}$ & $1,55 \mathrm{bc}$ & $16,47 \mathrm{a}$ \\
\hline $\mathrm{t} 2 \mathrm{xd} 2$ & $418,83 \mathrm{ab}$ & $1,51 \mathrm{bc}$ & $8,17 \mathrm{~b}$ \\
\hline $\mathrm{t} 2 \mathrm{xd} 3$ & $380,50 \mathrm{~b}$ & $1,62 \mathrm{bc}$ & $5,37 \mathrm{~d}$ \\
\hline $\mathrm{t} 3 \mathrm{xd} 1$ & $479,67 \mathrm{ab}$ & $1,79 \mathrm{abc}$ & $16,92 \mathrm{a}$ \\
\hline $\mathrm{t} 3 \mathrm{xd} 2$ & $441,17 \mathrm{ab}$ & $1,39 \mathrm{bc}$ & $6,95 \mathrm{bcd}$ \\
\hline $\mathrm{t} 3 \mathrm{xd} 3$ & $451,00 \mathrm{ab}$ & $1,98 \mathrm{ab}$ & $6,06 \mathrm{~cd}$ \\
\hline
\end{tabular}

*Medias con la misma letra no difieren estadísticamente, según la prueba de Tukey, $\alpha=0,05$, estadístico de prueba válido cuando la prueba $F$ resulta no significativa.

Los IAFs se ubicaron por encima de los valores de 8 , obtenidos bajo invernadero, reportados por Angulo (2005a). El IAF presentó una correlación negativa a un nivel de $\mathrm{P}<0,01$, con el volumen del fruto $(-0,48)$ y significó que los frutos a menor distancia de siembra fueron más pequeños y mayor área de suelo ocupada por las hojas comparativamente con los demás tratamientos. En Boyacá, Colombia, en cultivos sembrados a $1 \mathrm{x} 1 \mathrm{~m}$ y a una altura de $2.300 \mathrm{msnm}$, después de tres meses de plantación, alcanzaron un IAF de 14,5 a campo abierto, cercano al hallado en Subia y, a 2.690 msnm, lograron IAF de 3, después de nueve meses de cultivo; en ambas altitudes, el IAF fue de 12,5. El rápido desarrollo del IAF permitió, en forma temprana en Subia, obtener altas producciones de frutos, como lo encontraron Fischer et al. (2012).

Número de flores, volumen de fruto, peso del fruto sin cáliz y diámetro longitudinal del fruto: La floración de más del 50\% de las plantas, se determinó a los 77DDT y la máxi- ma de producción de flores, se presentó a los 106 DDT. En la tabla 4, se observa que el tratamiento con mayor número de flores fue $\mathrm{t} 2 \mathrm{xd} 2$, con $674,83 \mathrm{y} \mathrm{t} 1 \mathrm{xd} 3$, con 664,67 flores y se diferenciaron a un nivel de $\mathrm{P}<0,05$ de $\mathrm{t} 2 \mathrm{xd} 3$, con 575,50 . Los resultados indicaron que los tratamientos no siguieron la tendencia que se genera cuando hay un incremento en el espacio entre plantas, que es originar un mayor número de flores, tal como es reportado para la India, por Ali \& Singh (2014).

En la tabla 4, se observa que para el volumen del fruto los mejores tratamientos fueron $\mathrm{t} 3 \mathrm{xd} 3$, con $5,296 \mathrm{~cm}^{3}$; $\mathrm{t} 1 \mathrm{xd} 3$, con $5,199 \mathrm{~cm}^{3}$ y t2xd3, con $5,070 \mathrm{~cm}^{3}$, que se diferenciaron a un nivel de $\mathrm{P}<0,05$ de $\mathrm{t} 1 \mathrm{xd} 13,416$, t2xd1, con 3,399 y $\mathrm{t} 3 \mathrm{xd} 1$, con $3,285 \mathrm{~cm}^{3}$, que corresponden a los tratamientos con mayor rendimiento por hectárea, es decir, los volúmenes del fruto sonafectados por la densidad de siembra y disminuyen su peso en $\mathrm{t} 1 \mathrm{xd} 1, \mathrm{t} 2 \mathrm{xd} 1, \mathrm{t} 2 \mathrm{xd} 2 \mathrm{y} \mathrm{t} 3 \mathrm{xd} 2$, en mayor proporción, con relación a los otros tratamientos, que tienen 
distancias de siembra más amplias. Los valores de los mejores tratamientos fueron mayores a $4,340 \mathrm{~cm}^{3}$, hallado en la India, en condiciones de campo abierto, por Yadava (2012). El peso del fruto sin cáliz estuvo entre un rango comprendido entre 4,34 a 5,34g (Tabla 4), que se ubica dentro de los valores reportados en Colombia, bajo condiciones de invernadero, con cubierta plástica, en Pasto, Nariño, por Criollo et al. (2014). El volumen del fruto se correlacionó con el rendimiento por hectárea.
En la tabla 4 , se muestra que los mejores tratamientos para el diámetro longitudinal del fruto fueron $\mathrm{t} 3 \mathrm{xd} 3$, con $2,193 \mathrm{~cm}$; t2xd3, con $2,185 \mathrm{~cm}$ y t $1 \mathrm{xd} 3$, con $2,140 \mathrm{~cm}$, que se diferenciaron a un nivel de $\mathrm{p}<0,05$ de $\mathrm{t} 1 \mathrm{xd} 1$, con $1,900 \mathrm{~cm}$; t2xd1, con $1,847 \mathrm{~cm}$ y t3xd1, con $1,847 \mathrm{~cm}$; los tres últimos tratamientos corresponden a los de mayor rendimiento agronómico. Gasparetto et al. (2014) registraron valores de $1,986 \mathrm{~cm}$, para el diámetro longitudinal, en 15 frutos escogidos al azar, en el estado de maduración verde del fruto y

Tabla 4. Valores medios para las variables número total de flores, volumen fruto, peso fruto sin cáliz y diámetro longitudinal del fruto, en P. peruviana L.

\begin{tabular}{|c|c|c|c|c|}
\hline Tratamientos & Número total flores & Volumen fruto $(\mathrm{cm} 3)$ & Peso fruto sin cáliz $(\mathrm{g})$ & Diámetro longitudinal fruto $(\mathrm{cm})$ \\
\hline $\mathrm{t} 1 \mathrm{xd} 1$ & $632,00 \mathrm{ab} *$ & $3,416 \mathrm{c}$ & $4,93 \mathrm{a}$ & $1,900 \mathrm{~b}$ \\
\hline $\mathrm{t} 1 \mathrm{xd} 2$ & $649,17 \mathrm{ab}$ & $3,863 \mathrm{bc}$ & $5,34 \mathrm{a}$ & $1,978 \mathrm{ab}$ \\
\hline $\mathrm{t} 1 \mathrm{xd} 3$ & $664,67 \mathrm{a}$ & $5,199 \mathrm{a}$ & $5,12 \mathrm{a}$ & $2,140 \mathrm{a}$ \\
\hline $\mathrm{t} 2 \mathrm{xd} 1$ & $637,50 \mathrm{ab}$ & $3,399 \mathrm{c}$ & $4,51 \mathrm{a}$ & $1,847 \mathrm{~b}$ \\
\hline $\mathrm{t} 2 \mathrm{xd} 2$ & $674,83 \mathrm{a}$ & $4,132 \mathrm{abc}$ & $4,71 \mathrm{a}$ & $1,977 \mathrm{ab}$ \\
\hline $\mathrm{t} 2 \mathrm{xd} 3$ & $575,50 \mathrm{~b}$ & $5,070 \mathrm{ab}$ & $5,10 \mathrm{a}$ & $2,185 \mathrm{a}$ \\
\hline $\mathrm{t} 3 \mathrm{xd} 1$ & $634,50 \mathrm{ab}$ & $3,285 \mathrm{c}$ & $5,42 \mathrm{a}$ & $1,847 \mathrm{~b}$ \\
\hline $\mathrm{t} 3 \mathrm{xd} 2$ & $606,00 \mathrm{ab}$ & $4,085 \mathrm{abc}$ & $4,34 \mathrm{a}$ & $1,983 \mathrm{ab}$ \\
\hline $\mathrm{t} 3 \mathrm{xd} 3$ & $648,60 \mathrm{ab}$ & $5,296 \mathrm{a}$ & $5,10 \mathrm{a}$ & $2,193 \mathrm{a}$ \\
\hline
\end{tabular}

*Medias con la misma letra no difieren estadísticamente, según la prueba de Tukey, $\alpha=0,05$, estadístico de prueba válido cuando la prueba $F$ resulta no significativa.

cáliz, bajo condiciones de invernadero, en Brasil; ese valor esmenor a los mejores encontrados en Subia; también hallaron que el diámetro se incrementa a medida que el fruto madura fisiológicamente.

Almendagna et al. (2012) encontraron, en Brasil, un valor de $1,855 \mathrm{~cm}$, para el diámetro longitudinal en frutos cosechados con cáliz amarillo de plantas de cinco meses edad, que fueron cultivadas en casa de vegetación; valores menores a los hallados en Subia. Un estudio de Muniz et al. (2011), también en Brasil, reveló diámetros menores a los de Subia, usando cuatro tipos de tutorados, siendo el peor el $1,365 \mathrm{~cm}$, hallado en el sistema de espaldera.considerablemente menor al tratamiento en Subia, que fue $\mathrm{t} 3 \mathrm{xd} 1$, con $1,847 \mathrm{~cm}$. Los rangos encontrados en el presente estudio, para el diámetro, estuvieron ubicados entre 1,847 a $2,193 \mathrm{~cm}$ y son mayores a los mostrados con frutos provenientes de campo abierto, del municipio de Granada, Cundinamarca (Colombia), por Borda \& Caicedo (2013).
Los frutos pequeños obtenidos con $\mathrm{t} 1 \mathrm{xd} 1$ son antieconómicos, en caso de ser utilizados para la extracción de jugos, ya que es necesario un mayor número de frutos, como lo afirman Almendagna et al. (2012). Los mejores tratamientos $\mathrm{t} 3 \mathrm{xd} 3, \mathrm{t} 2 \mathrm{xd} 3, \mathrm{t} 1 \mathrm{xd} 3, \mathrm{t} 1 \mathrm{xd} 2, \mathrm{t} 3 \mathrm{xd} 2, \mathrm{t} 2 \mathrm{xd} 2$, para el diámetro longitudinal de frutos, pueden ser más atractivos para el consumidor, como lo aseveran Muniz et al. (2011) y, según el Instituto Colombiano de Normas Técnicas (1999), se ubicarían en las clases $\mathrm{B}$ y $\mathrm{C}$.

Número de frutos, rendimiento por planta, rendimiento por hectárea y sólidos solubles totales (azúcares), expresada en ${ }^{\circ}$ Brix: La tabla 5 muestra que los mejores tratamientos para número de frutos fueron $\mathrm{t} 1 \mathrm{xd} 2 \mathrm{y} \mathrm{t} 1 \mathrm{xd} 3$, con 7205,8 y 6989,3 frutos, respectivamente y se diferenciaron a un nivel de $\mathrm{P}<0,05$ de $\mathrm{t} 2 \mathrm{xd} 3$, con 4973,7. Mora-Aguilar et al. (2006) establecieron que el número de frutos correlacionó positivamente con el rendimiento por hectárea, en un cultivo establecido bajo invernadero, en México. En Subia, también se correlacionó con altura de planta $(0,65)$ y número de ramas 
Tabla 5. Valores medios para las variables número de frutos, rendimiento por planta, rendimiento y sólidos solubles totales (azúcares) expresada en ${ }^{\circ}$ Brix, en $P$. peruviana L.

\begin{tabular}{|c|c|c|c|c|}
\hline Tratamientos & $\begin{array}{l}\text { Numero de frutos totales } \\
\text { cosechados }\end{array}$ & $\begin{array}{l}\text { Rendimiento por } \\
\operatorname{planta(g)}\end{array}$ & Rendimiento (t.ha ${ }^{-1}$ ) & Grados ${ }^{\circ}$ Brix \\
\hline $\mathrm{t} 1 \mathrm{xd} 1$ & $6084 a b^{*}$ & $460,42 \mathrm{ab}$ & $27,704 \mathrm{a}$ & $16,01 a$ \\
\hline $\mathrm{t} 1 \mathrm{xd} 2$ & $7205,8 a$ & $574,50 \mathrm{a}$ & $15,412 b$ & $15,93 a$ \\
\hline $\mathrm{t} 1 \mathrm{xd} 3$ & $6989,3 a$ & 552,67 a & $11,106 \mathrm{~cd}$ & $16,13 a$ \\
\hline $\mathrm{t} 2 \mathrm{xd} 1$ & $6076,5 \mathrm{ab}$ & $427,92 b$ & 25,781 a & $16,06 a$ \\
\hline $\mathrm{t} 2 \mathrm{xd} 2$ & $5771,3 a b$ & $390,17 b$ & $12,638 b c$ & $16,28 a$ \\
\hline $\mathrm{t} 2 \mathrm{xd} 3$ & $4973,7 b$ & $341,50 b$ & $7,929 d$ & $15,83 a$ \\
\hline $\mathrm{t} 3 \mathrm{xd} 1$ & $6255 a b$ & $352,08 b$ & $27,577 \mathrm{a}$ & $15,93 a$ \\
\hline $\mathrm{t} 3 \mathrm{xd} 2$ & $5974,5 a b$ & $379,50 b$ & $13,062 b c$ & $16,08 a$ \\
\hline $\mathrm{t} 3 \mathrm{xd} 3$ & $6635,7 a b$ & $406,67 b$ & 9,530cd & $16,23 a$ \\
\hline
\end{tabular}

*Medias con la misma letra no difieren estadísticamente, según la prueba de Tukey, $\alpha=0,05$, estadístico de prueba válido cuando la prueba $\mathrm{F}$ resulta no significativa.

$(0,61)$, lo que indica que el área de la planta, donde se puede mantener flores y frutos cuajados, después de la antesis, son determinantes para obtener buenos rendimientos.

Los mejores tratamientos para el rendimiento fueron $\mathrm{t} 1 \mathrm{xd} 1$, con 27,704t.ha ${ }^{-1}$; t3xd1, con 27,577t.ha ${ }^{-1}$ y t2xd1, con 25,781 t.ha ${ }^{-1}$, presentaron diferencias a un nivel de $\mathrm{P}<0,05$ con t1xd2, con 15,412t.ha ${ }^{-1}$; t3xd2, con 13,062t.ha ${ }^{-1}$; t2xd2, con 12,638t.ha 1; t1xd3, con 11,106t.ha ${ }^{-1}$; t3xd3, con 9,530t. ha-1 y t2xd3, con 7,929t.ha ${ }^{-1}$ (Tabla 5), rendimientos logrados durante 305 días del ciclo del cultivo, con una duración de la época de cosecha de seis meses y mayor al reportado para Brasil, por Rufato et al. (2008). Los rendimientos agronómicos de $\mathrm{t} 1 \mathrm{xd} 1$, $\mathrm{t} 3 \mathrm{xd} 1 \mathrm{y} \mathrm{t} 2 \mathrm{xd} 1$ obtenidos fueron mayores a los 12,000t.ha ${ }^{-1}$, utilizando el tutorado en $\mathrm{T}$ y una distancia de siembra de $3 \times 2 \mathrm{~m}$, en Chile, por Cruzat \& Honorato, (2010). Los mejores tratamientos para el rendimiento por hectárea, se encontraron dentro del rango de 15 a 28t.ha ${ }^{-1}$, reportado por Miranda (2004). El rendimiento por hectárea estuvo correlacionado con el número de ramas $(0,50)$, IAF $(0,54)$ y ${ }^{\circ}$ Brix $(0,54)$ mostrando que un determinado número de ramas, IAF y unos sólidos solubles totales determinan el aumento del rendimiento, lo que sugiere que la intercepción de la luz es crucial para obtener buen rendimiento por hectárea y niveles comercializables de sólidos solubles totales.

Los valores obtenidos con el sistema tipo espaldera fueron mayores a los registrados en Brasil, con los sistemas "V" invertido, vertical y triangular, los cuales, evidenciaron rendimientos de 9,720 t. ha ${ }^{-1}, 4,530$ t.ha ${ }^{-1}$ y 8,540t.ha ${ }^{-1}$, respectivamente (Muniz et al. 2011). Los mejores tratamientos para el rendimiento por planta fueron $\mathrm{t} 1 \mathrm{xd} 1$, con $460,42 \mathrm{~g}$; t1xd2, con $574,50 \mathrm{~g}$ y $\mathrm{t} 1 \mathrm{xd} 3$, con $552,67 \mathrm{~g}$ y se diferenciaron a un nivel de $\mathrm{P}<0,05$ con $\mathrm{t} 2 \mathrm{xd} 3$, con $341,50 \mathrm{~g}$ y $\mathrm{t} 3 \mathrm{xd} 1$, con $352,08 \mathrm{~g}$. El mejor rendimiento en $\mathrm{t} 1 \mathrm{xd} 1$, se explica, porque este tratamiento permite un mejor crecimiento, desarrollo, aireación y penetración de la luz, como lo afirman Lima et al. (2010). En la tabla 5, se observa que los sólidos solubles totales, expresados como ${ }^{\circ}$ Brix, no presentaron diferencias significativas a un nivel de $\mathrm{P}<0,05$. Los mayores ${ }^{\circ}$ Brix fueron observados en $\mathrm{t} 2 \mathrm{xd} 2$, con $16,28^{\circ}$ Brix; t3xd3, con $16,23^{\circ}$ Brix y t1xd3, con $16,13^{\circ}$ Brix y los menores: $\mathrm{t} 3 \mathrm{xd} 1$, con $15,93^{\circ} \mathrm{Brix}$ y $\mathrm{t} 2 \mathrm{xd} 3$, con $15,83^{\circ}$ Brix. Los tratamientos con mayor rendimiento agronómico tendieron a tener menos ${ }^{\circ}$ Brix, volumen del fruto y peso del fruto, como se observa en las tablas 4 y 5 .

De acuerdo con las normas de la comisión del Codex Alimentarius (2007), los frutos deben presentar un mínimo de $14^{\circ}$ Brix para ser comercializados en Colombia; por lo tanto, los frutos producidos en Subia presentaron valores de sólidos solubles totales por encima del mínimo para su mercadeo. En Brasil, en los sistemas de conducción en "V", "X" e espaldera, se encontró que valores observados de sólidos solubles totales fueron de 9,23 "Brix, en el sistema " $X$ " y $9,93^{\circ}$ Brix, sin usar tutor (Muniz et al. 2011). De acuerdo a todo lo anterior, se puede concluir que el tipo chiquero (t1) y la menor distancia de siembra $(\mathrm{d} 1: 1 \mathrm{x} 2 \mathrm{~m})$ fue la mejor combinación, que permitió una mejor distribución de las ramas en las plantas y área foliar que interceptó la luz solar, lo cual, redundó en un mejor desarrollo de las mismas, en términos de altura, número de ramas, número de flores, número de frutos totales y rendimiento por hectárea. 
Financiación: Este estudio fue financiado por la Universidad de Cundinamarca, la comunidad de la vereda el Pedregal en Subia y los autores. Conflictos de intereses: El manuscrito fue preparado y revisado con la participación de todos los autores, quienes declaramos que no existe ningún conflicto de intereses que ponga en riesgo la validez de los resultados presentados.

\section{BIBLIOGRAFÍA}

1. ALI, A.; SINGH, B.P. 2014. Plant spacing and NPK fertilizers affecting flowering fruiting of Cape gooseberry (P. peruviana L.) grown in sodic soil. J. Environm. Ecol. (India). 32(2A):767-771.

2. ANGULO, R. 2005. Crecimiento, desarrollo y producción de la uchuva en condiciones de invernadero y campo abierto. En: Fischer, G. (ed.). Avances en cultivo, poscosecha y exportación de la uchuva ( $P$. peruviana L.) en Colombia. U. N. Colombia, Fac. Agronomía, Bogotá D.C. p.111-130.

3. ALMENDAGNA, F.R.; PENONI, E. DOS SANTOS; RODRIGUES, J.D.S.; PASQUAL, M. 2012. Caracterização do ponto de colheita de $P$. peruviana $\mathrm{L}$. Na região de Lavras, MG. Rev. Biosci. J. Uberlândia. (Brasil) 28(6):862-867.

4. BORDA, C.; CAICEDO, O. 2013. Cambios en el contenido nutricional de la uchuva (P. peruviana) frente a osmodeshidratación como método de conservación. (Colombia) Rev. Perspect. Nutr. Humana. 15:149156.

5. COMISIÓN DEL CODEX ALIMENTARIUS. 2007. Norma del CODEX para la Uchuva. En: Organización Mundial de la Salud y Organización de las Naciones Unidas para la Agricultura y la Alimentación (eds). Frutas y Hortalizas Frescas. p.131-135. Disponible desde internet en: ftp://ftp.fao.org/codex/publications/Booklets/FreshFruitsVeg/FFV_2007_ES.pdf (con acceso 11/08/2014).

6. CRIOLLO, H.; LAGOS, T.C.; FISCHER, G.; MORA, L.; ZAMUDIO, L. 2014. Comportamiento de tres genotipos de uchuva (P. peruviana L.) bajo diferentes sistemas de poda. Rev. Col. Cienc. Hortíc. 8(1):34-43.

7. CRUZAT, G.R.; HONORATO, G.C. 2010. Resultados y lecciones en cultivo de goldenberry (P. peruviana L.) en la zona central de Chile. Proyecto de Innovación en Región del Maule. Fundación para la Innovación Agraria. Min. Agri. Frutales/Berries 90, serie expe- riencias de innovación para el emprendimiento agrario. 58p.

8. FISCHER, G.; ALMANZA, P.J.M.; MIRANDA, D. 2014. Importancia y cultivo de la uchuva (P. peruviana L.). Rev. Bras. Frutic., Jaboticabal - SP. (Brasil). 36(1):115.

9. FISCHER, G.; ALMANZA, P. J. M.; RAMÍREZ, F. 2012. Source-sink relationships in fruit species: A review. Rev. Col. Cienc. Hortíc. 6(2):238-253.

10. FISCHER, G.; HERRERA, A.; ALMANZA, P.J.M. 2011. Cape gooseberry (P. peruviana L.). En: Yahia, E.M. (ed.) Postharvesth biology and technology of tropical fruits. Vol. 2. Acai to citrus. Woodhead Publishing, Cambridge, UK. p.374-396.

11. FISCHER, G.; MIRANDA, D. 2012. Uchuva (P. peruviana L.). En: Fischer, G. (eds). Manual para el cultivo de frutales en el trópico. Produmedios. Bogotá D.C. p.851-873.

12. GASPARETTO, C.A.S.; ZUCARELI, C.; MATEUS, A.P.; VICENTIN DE ALMEIDA, B.B. DA SILVA. 2014. Maturation stages of fruit development and physiological seed quality in P. peruviana. Rev. Ciênc. Agron. (Brasil) 45(3):543-549.

13. INSTITUTO COLOMBIANO DE NORMAS TÉCNICAS Y CERTIFICACIÓN-ICONTEC. 1999. Norma técnica colombiana uchuva NTC 4580. Bogotá D.C. 15p.

14. LIMA, C.S.M.; GONÇALVES, M.A.; FONSECA, Z.P.T.; RUFATO, A. de R.; FACHINELLO, J.C. 2010. Sistemas de tutoramento e épocas de trasplante de Physalis. Rev. Ciênc. Rural. (Brasil) 40(12):2472-2479.

15. LIMA, C.S.M; SEVERO, J.; MANICA-BERTO, R.; PROCATH DA SILVA, S.J.; LEITZKE, D.B.; RUFATO, A. DE R. 2009. Custos de implantação e condução de pomar de Physalis na região sul do estado do Rio Grande do Sul. Rev. Ceres, Viçosa. (Brasil) 56(5):555561.

16. MIRANDA, D. 2004. Informes de visitas de asesoría técnica a fincas productoras de uchuva (P. peruviana L.) en la Sabana de Bogotá y Antioquia. Fac. de Agronomía, U. N. Colombia, Bogotá D.C. 35p.

17. MIRANDA, D. 2005. Criterios para el establecimiento, los sistemas de cultivo, el tutorado y la poda de la uchuva. En: Fischer, G.; Miranda, D.; Piedrahíta, W.; 
Romero, J. (eds). Avances en cultivo, poscosecha y exportación de la Uchuva P. peruviana L. en Colombia. U. N. Colombia, Fac., Bogotá D.C. p.29-54.

18. MORA-AGUILAR, R.; PEÑA-LOMELÍ, A.; LÓPEZ-GAYTÁN, E.; AYALA-HERNÁNDEZ, J.J.; PONCE AGUIRRE, D. 2006. Agrofenología de P. peruviana L. en invernadero y fertirriego. Rev. Chapingo Ser. Hortic. (México) 12(1):57-63.

19. MUNIZ, J.; KRETZSCHMAR, A.A.; RUFATO, L.; PELIZZA, T.R.; MARCHI, T.; EUSÉBIO, A.D.; FERNANDES, L.A.P.; GARANHANI, F. 2011. Sistemas de condução para o cultivo de Physalis no Planalto Catarinense. Rev. Bras. Frut., Jaboticabal - SP (Brasil). 33(3):830838.

20. MUNIZ, J.; KRETZSCHMAR, A. A.; RUFATO, L.; PELIZZA, T R.; RUFATO, A. de R.; DE MACEDO, T.A. 2014. General aspects of Physalis cultivation. Ciênc. Rural, Santa Maria (Brasil). 44(6):964-970.

21. PANAYOTOV, N.; POPOVA, A. 2014. Vegetative Behaviors of Cape Gooseberry (P. peruviana L.) Grown by Direct Sowing Outside Under Conditions of Bulgaria. Turkish J. Agr. Nat. Sci. Special ISSUE 1:1141-1146.

22. PANAYOTOV, N.; POPOVA, A. 2013. Effect of different rates of nitrogen fertilization on vegetative development and productivity of Cape gooseberry $(P$. peruviana L.). IN: D'Haene, K.; Van de Casteele, B.; De Vis, R.; Crappé, S.; Callens, D., Mechant, E.; Hofman, G.; De Neve, S. (Eds) Nutrihort: Nutrient management, innovative techniques and nutrient legislation in intensive horticulture for an improved water quality. Institute for Agricultural and Fisheries Research (Ghent, Belgium). p.350-357.
23. PEIXOTO, N.; PEIXOTO, F.C.; VAZ, U.L.; NERI, S.C.M.; MONTEIRO, J.G. 2010. Adubação orgânica e cobertura do solo no crescimento e produção de camapu. Rev. Hortic. Bras. 28:370-372.

24. RAMÍREZ, F.; FISCHER, G.; THOMAS, LEE D. T.; PINZÓN, J.C.A.; ULRICHS, C. 2013. Cape gooseberry (P. peruviana L.) phenology according to the $\mathrm{BBCH}$ phenological scale. Sci. Hort. (Holland) 162:39-42.

25. RUFATO, L.; RUFATO, A. de R.; SCHLEMPER, C.; LIMA, C.S.M.; KRETZSCHMAR, A.A. 2008. Aspectos técnicos da cultura da Physalis. 1. ed. Lages: CAV/ UDESC; Pelotas: UFPel. 100p.

26. SALAZAR, M.R.; JONES, J.W.; CHAVES, B.; COOMAN, A. 2008. A model for the potential production and dry matter distribution of Cape gooseberry ( $P$. peruviana L.). Sci. Hort. 115:142-148.

27. TORRES, J.F.R.; GÓMEZ, E.C.; GROMA, A; BONO, M.; ESPAÑA, B.P.; SAN BAUTISTA, A.P.; ALARGADA, J.P.; LÓPEZ, S.G.; MAROTE, J.V.B. 2014. Uchuva o alquenquenje (P. peruviana L.). Rev. Agrícola Vergel: Fruticultura, horticultura, floricultura (España). 33(372):56-63.

28. YADAVA, L. P. 2012. Effect of growth retardants on floral biology, fruit set and fruit quality of Cape Gooseberry (P. peruviana L.). Am. J. Plant. Physiol. (USA). 7(3):143-148.

Recibido: Junio 22 de 2014

Aceptado: Febrero 15 de 2015

Cómo citar:

Quevedo García, E.; Sánchez García, O.; Veloza Sandoval, C.E. 2015. Efecto del tutorado y distancias de siembra sobre el rendimiento de Physalis peruviana L. Rev. U.D.C.A Act. \& Div. Cient. 18(1): 91-99. 